\title{
Editorial
}

\section{Preface to Special Issue on Advances in Ceria Catalysis}

Ceria, which acts as a "star" among the metal-oxides, has established to be one of the most promising materials for chemical transformations and energy applications owing to its redox and acid-base properties. Ceria has become a key component in three-way catalytic converters, water-gas shift reaction, CO oxidation, methane combustion, and complex organic reactions. More importantly, introducing of other metals to ceria, tailoring the shape of the ceria, and reducing structure size of ceria, usually decrease the formation energy of oxygen vacancy (Vö) and lead to unique catalytic performances.

With the rapid development in catalyst synthesis methods and characterization techniques, ceria-based catalysts with specific active sites are readily obtainable, providing new opportunities to produce catalysts with excellent activity, selectivity and good stability to realize energetically challenging reactions, unlock the reaction mechanism, and explore the potential applications in new organic reactions and industrial production. To date, the development of ceria catalysis has achieved noticeable advances, which motivates us to put together a special issue dedicated to "Advance in Ceria Catalysis". This special issue contains 12 invited papers including 5 reviews, 1 communication, and 6 articles, covering the most inprotant aspects of structure-activity inverstigation, methanism exploration, and new orginic reactions. In the following, a brief description of the papers included in this issue is provided to serve as an outline to encourage further reading.

The rational design and construction of ceria-based catalysts with specific active sites as well as the structure-activity investigation are of great importance to achieve excellent performances for chemical transformations such as selective hydrogenation of alkynes, methane combustion, $\mathrm{CO}$ oxidation, $\mathrm{CO}_{2}$ hydrogenation, and so on. The review article by Wu et al. summarized recent advances in both experimental and computational studies of ceria, focusing on the interaction of ceria with $\mathrm{H}_{2}$ and the applications to selective hydrogenation of alkynes. Ways to further improve both the mechanistic understanding and catalytic performance of ceria-based materials for hydrogenation reactions are also proposed. The review article by Perez-Ramirez et al. provided an overview of recently developed ceria-based catalysts in the context of polymer industry (polyvinyl chloride, polyurethanes, and polycarbonates) and activation of light hydrocarbons for natural gas upgrading. The review article by Shen et al. demonstrated that both the shape of ceria and the loading of copper affect the chemical bonding of copper species on ceria surfaces and the electronic and geometric characters of the relevant interfaces. The review article by Trovarelli et al. summarized the latest findings in methane combustion catalyzed by $\mathrm{Pd}-\mathrm{CeO}_{2}$, and particularly to envisage how the control (when possible) of $\mathrm{Pd}_{-} \mathrm{CeO}_{2}$ interaction at nanoscale can help in designing more robust methane combustion catalysts. Janik and Rioux et al. reviewed the oxidation of $\mathrm{CO}$ w/o the presence of hydrogen over ceria-supported metal catalysts with focus on the impact of metal nuclearity on the reaction performances and mechanisms. Cargnello et al. developed a nanoparticle-based technique that allows for quantification of the capacity of a support to stabilize single atoms.by re-dispersing $\mathrm{Ru}$ nanoparticles into single atoms and taking advantage of the different catalytic properties of $\mathrm{Ru}$ single atoms and nanoparticles in the $\mathrm{CO}_{2}$ hydrogenation reaction. Huang et al. investigated the structures and catalytic activity in CO oxidation of $\mathrm{CuO}_{x}$ species on $\mathrm{CeO}_{2}$ rods, cubes and polyhedra predominantly exposing respectively $\{110\}+\{100\}$, $\{100\}$ and $\{111\}$ facets. They comprehensively elucidated the $\mathrm{CuO}_{x}-\mathrm{CeO}_{2}$ interactions and exemplified their morphology-dependence. Jia et al. deposited $1 \mathrm{wt} \%$ of copper on $\mathrm{CeO}_{2}$ nanocubes mainly exposing $\{100\}$ planes (1CuCe NC) and nanorods (1CuCe NR) exposing high contents of $\{110\}$ facets, respectively. The excellent redox properties and effective $\mathrm{CO}$ adsorption induce the greatly enhanced catalytic reactivity for the $1 \mathrm{CuCe} \mathrm{NC}$ catalyst. The rational design and construction of catalysts with specific active sites is of great importance to achieve excellent performances for chemical transformations.

Exploring new applications in orgonic reactions is of great importance for ceria-based solid catalysts. Wang et al. synthesized three catalysts including $\mathrm{Ru}$-clusters $/ \mathrm{CeO}_{2}$, 
$\mathrm{Ru} / \mathrm{CeO}_{2}$-rod, and $\mathrm{Ru} / \mathrm{CeO}_{2}$-cube for hydromethoxycarbonylation reaction of styrene. Ru-clusters/ $\mathrm{CeO}_{2}$ showed the best catalytic performance for this reaction with > 99\% conversion of styrene, giving 83\% and 12\% regioselectivity of linear and branched ester, respectively. Shimizu et al. reported that a commercially available $\mathrm{CeO}_{2}$ is an active and reusable catalyst for the $\mathrm{C}_{3}$-selective alkenylation of oxindole with aldehydes under solvent-free conditions. The structure-activity relationship study using $\mathrm{CeO}_{2}$ catalysts with different sizes suggests that defect-free $\mathrm{CeO}_{2}$ surface is the active site for this reaction.

Model studies on ceria catalysis have provided deep insights into the interfacial structure of metal-oxide down to the atomic level, and have shed light onto many catalytic processes. Hensen et al. investigated the influence of doping transition metal (TM) ions into the ceria surface on the activation of surface lattice oxygen atoms via density functional theory (DFT) calculations. The findings emphasize the origin of lattice oxygen activation and the preferred location of TM dopants in TM-ceria solid solution catalysts. Libuda et al. prepared and characterized atomically well-defined model systems for ceria-supported Pt-Co core-shell catalysts and the structural evolution as a function of temperature.
As guest editors, we would like to thank all the authors who contributed to this issue. Their contributions represent excellent examples of the current research trends in the field of ceria catalysis. We also wish to thank the editorial staff of Chinese Journal of Catalysis for their help to organize this issue. We hope that the topics presented in this issue would inspire the readers to further explore the interesting science of ceria catalysis.

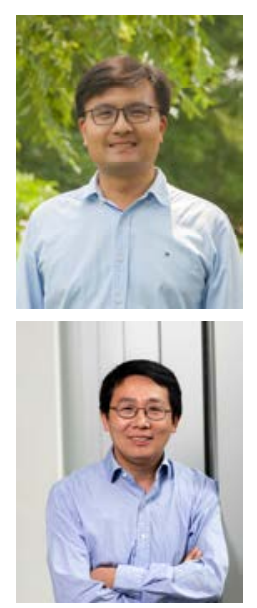

Professor Feng Wang

Lab of Bioenergy Chemical

Dalian Institute of Chemical Physics, CAS,

China

E-mail: wangfeng@dicp.ac.cn

Dr. Zili Wu

Chemical Science Division and Center for Nanophase Materials Sciences Oak Ridge National Laboratory, USA

E-mail: wuz1@ornl.gov 